\title{
Evaluation of platelet-rich plasma from diabetic donors shows increased platelet vascular endothelial growth factor release
}

\author{
Karina $^{1,2,3}$, Komang Ardi Wahyuningsih ${ }^{2,4,}$ Siti Sobariah $^{2}$, Iis Rosliana ${ }^{2}$, Imam Rosadi ${ }^{2}$, Tias Widyastuti', \\ Irsyah Afini ${ }^{2}$, Septelia Inawati Wanandi ${ }^{5,6}$, Pradana Soewondo ${ }^{7}$, Heri Wibowo ${ }^{8}$, Jeanne Adiwinata Pawitan ${ }^{9,10,11}$ \\ ${ }^{1}$ Doctoral Program of Biomedical Sciences, Faculty of Medicine, Universitas Indonesia, Jakarta, Indonesia; ${ }^{2}$ Hayandra Lab, ${ }^{3}$ Klinik Hayandra, \\ Yayasan Hayandra Peduli, Jakarta, Indonesia; ${ }^{4}$ Department of Histology, Universitas Katolik Indonesia Atma Jaya, Jakarta, Indonesia; ${ }^{5}$ Department \\ of Biochemistry and Molecular Biology, Faculty of Medicine, ${ }^{6}$ Molecular Biology and Proteomics Core Facilities, Indonesian Medical Education and \\ Research Institute, Faculty of Medicine, ${ }^{7}$ Division of Metabolism and Endocrinology, Department of Internal Medicine, Dr. Cipto Mangunkusumo \\ Hospital, Faculty of Medicine, ${ }^{8}$ Laboratorium Terpadu, Faculty of Medicine, ${ }^{9}$ Department of Histology, Faculty of Medicine, ${ }^{10}$ Stem Cell Medical \\ Technology Integrated Service Unit, Cipto Mangunkusumo Central Hospital, Faculty of Medicine, ${ }^{11}$ Stem Cell and Tissue Engineering Research \\ Center, Indonesia Medical Education and Research Institute (IMERI), Faculty of Medicine, Universitas Indonesia, Jakarta, Indonesia \\ Contributions: (I) Conception and design: Karina, JA Pawitan, SI Wanandi, P Soewondo; (II) Administrative support: None; (III) Provision of study \\ material or patients: Karina; (IV) Collection and assembly of data: I Rosadi, I Rosliana, I Afini, T Widyastuti, S Sobariah; (V) Data analysis and \\ interpretation: Karina, I Rosliana, JA Pawitan, SI Wanandi, P Soewondo, H Wibowo, KA Wahyuningsih; (VI) Manuscript writing: All authors; (VII) \\ Final approval of manuscript: All authors. \\ Correspondence to: Karina. Doctoral Program of Biomedical Sciences, Faculty of Medicine, Universitas Indonesia, Jakarta, Indonesia; Hayandra Lab, \\ Yayasan Hayandra Peduli, Jakarta, Indonesia; Klinik Hayandra, Yayasan Hayandra Peduli, Jakarta, Indonesia. Email: karina@hayandra.com.
}

Background: Platelet-rich plasma (PRP) contains pro-angiogenic growth factors including vascular endothelial growth factor (VEGF). Angiogenesis is a necessary component of wound healing in instances of diabetic foot ulcers (DFU). PRP composition varies depending on methods and donor health status. Our group has developed an improved PRP protocol for diabetes treatment. The aims of this study were to examine the levels of the pro-angiogenic factor VEGF in these patient populations with and without diabetes.

Methods: PRP was prepared using $24 \mathrm{~mL}$ of whole blood from 13 diabetic and 10 non-diabetic patients registered at Klinik Hayandra. Whole blood in sodium citrate tubes were centrifuged at 1,000 rpm for 5 minutes followed by plasma separation. Plasma samples were centrifuged at 3,000 rpm for 5 minutes. Upper platelet-poor plasma layers were discarded, leaving $5 \mathrm{~mL}$ of concentrated platelet containing plasma (PRP). Concentrated plasma samples were mixed, aliquoted, stored at $-86^{\circ} \mathrm{C}$, and pooled for platelet count, VEGF, and total protein analyses. Platelet counting was also performed using fresh whole blood and PRP to measure changes following PRP preparation.

Results: Diabetic donors had higher whole blood platelet counts than non-diabetic donors, but this difference was not statistically significant. An average increase of more than $250 \%$ in platelet number after PRP preparation using our method was noted in both groups. Freezing-thawing samples at $-86{ }^{\circ} \mathrm{C}$ lysed more than $90 \%$ of PRP platelets regardless of diabetes status. Diabetic PRP had lower mean total protein and higher VEGF concentrations. Lysed platelets from diabetic donors released more VEGF than those from non-diabetic donors.

Conclusions: PRP from diabetic donors had higher VEGF content making autologous PRP application a promising treatment for DFU. However, this should be investigated another appropriate clinical trial.

Keywords: Angiogenesis; diabetes; diabetic foot ulcer (DFU); platelet-rich plasma (PRP); vascular endothelial growth factor (VEGF)

Received: 15 July 2019; Accepted: 06 September 2019; Published: 20 December 2019.

doi: $10.21037 /$ sci.2019.10.02

View this article at: http://dx.doi.org/10.21037/sci.2019.10.02 


\section{Introduction}

Platelet-rich plasma (PRP) is the plasma fraction containing many growth factors, prepared from whole blood samples. PRP has been used for the treatment of many diseases including general wounds (1), diabetic foot ulcers (DFU) (2), Crohn's disease (3), psoriasis (4), degenerative musculoskeletal diseases (5), and in cosmetic applications (6). In-vitro studies have demonstrated the efficacy of PRP in enhancing the proliferation of numerous cell types including endothelial cells (7), fibroblasts (8), keratinocytes (8), and mesenchymal stem cells (9). Particularly in mesenchymal stem cells culture applications PRP has been widely used as a replacement for fetal bovine serum or fetal calf serum in the culture media. Due to risks of animal to human transmission of disease or immunogenetic concerns when using animal serum in clinical cell culture applications use of non-animal origin material (10), such as PRP, is preferred.

Diabetes prevalence in Indonesia has been on the rise since 1983. DFU is one of the most commonly reported complications of diabetes. A previous study has reported that $7.3-24 \%$ of diabetic patients had DFU. Because the prevalence of undiagnosed diabetes is higher than what is reported the actual prevalence of DFU is expected to be under reported (11).

Patients who develop DFU experience decreased quality of life through increased medically associated costs of consultation, hospitalization, and surgery for treatment. DFU management should focus on providing early diagnosis and wound treatment in order to mitigate amputation risks. Current standards in DFU management are wound debridement, diagnosis and treatment of infection, revascularization and ulcer off-loading (11). However, there were still about $41 \%$ of DFU patients studied at the Tertiary Care Hospital in Semarang, Indonesia, the required lower extremity amputation. These data indicate that there is a need for developing new strategies of DFU management.

PRP has been used in several clinical settings for treating diabetic ulcer. However, there are multiple PRP preparation protocols that affect the quality of the PRP itself. Several studies have investigated the plasma or serum composition of diabetic and non-diabetic patients $(12,13)$. However, the preparation technique used in these studies were different from the protocol we have developed. In this study, the quality of PRP from diabetic patients produced by our protocol was evaluated and reported based on total platelet counts, vascular endothelial growth factor (VEGF) and protein levels, and were compared to PRP from non- diabetic patients.

\section{Methods}

\section{Patient population}

Thirteen diabetic patients and ten non-diabetic male and female patients aged from 25 to 75 years old registered at the Klinik Hayandra, with no hematological disease were enrolled in this study. Diabetic donors had disease duration of less than 5 years and were taking medication to control blood glucose levels. Non-diabetic donors had blood HbA1c levels less than $6.5 \%$. The study was conducted in accordance with protocols reviewed and approved by Health Research Ethics Committee, University of Indonesia and Cipto Mangunkusumo Hospital with issued letter of approval No. 628/UN2.F1/ETIK/2016.

\section{PRP preparation}

PRP was prepared using a standard protocol. Briefly, blood was collected into four sodium citrate tubes. Tubes were centrifuged at $1,000 \mathrm{rpm}$ for 5 minutes to separate plasma and red blood cells. Whole plasma from each tube was removed and collected into a single $15 \mathrm{~mL}$ conical tube per patient. Samples were then centrifuged at 3,000 rpm for 5 minutes resulting in concentrating platelets at the bottom of the tube. The upper platelet poor plasma (PPP) layer was discarded and the remaining $5 \mathrm{~mL}$ of PRP was collected and samples were stored at $-86^{\circ} \mathrm{C}$.

\section{Blood cell counts and protein analysis}

Blood cell counts using $500 \mu \mathrm{L}$ of whole blood or prepared PRP were performed using hematology analyzer (Sysmex KX 21, Sysmex America, Inc, USA). Prior to analysis, frozen PRP was thawed, then platelet number was counted. VEGF levels were quantified using enzyme-linked immunosorbent assay (ELISA) as described by the manufacturer (Human VEGF Quantikine ELISA Kit \#DVE00, R\&D Systems Europe, Abingdon, UK). Total protein in PRP was quantified through colorimetric assay (NanoDrop ${ }^{\mathrm{TM}}$ One Micro volume UV-Vis Spectrophotometers, Thermo Fisher Scientific, Wilmington, USA) following manufacturers protocol using the Pierce ${ }^{\mathrm{TM}}$ BCA Protein Assay Kit \#23227 (Thermo Scientific, Rockford, USA). VEGF level were presented in $\mathrm{pg} / \mathrm{mL}, \mathrm{pg} / \mathrm{mg}$ total protein, and $\mathrm{fg} / 1 \times 10^{3}$ lysed platelets. The levels of VEGF released from platelets 
Table 1 Donor demographics

\begin{tabular}{lccc}
\hline Parameter & Non-diabetic group & Diabetic group & P value \\
\hline Sample size $(\mathrm{n})$ & 10 & 13 & - \\
Male & $7(70 \%)$ & $8(62 \%)$ & - \\
Female & $3(30 \%)$ & $5(38 \%)$ & - \\
Age (years) & $61 \pm 10$ & $61 \pm 14$ & 0.83 \\
HbA1c (\%) & $5.58 \pm 4.67$ & $7.06 \pm 1.30$ & 0.001 \\
\hline
\end{tabular}

Table 2 Platelet-rich plasma composition of diabetic and nondiabetic donors

\begin{tabular}{|c|c|c|c|}
\hline Parameter & $\begin{array}{l}\text { Non-diabetic } \\
\text { group }\end{array}$ & Diabetic group & $P$ value \\
\hline $\begin{array}{l}\text { Whole blood platelet } \\
\left(\times 10^{3} / \mu \mathrm{L}\right)\end{array}$ & $201.40 \pm 66.18$ & $220.19 \pm 95.69$ & 0.60 \\
\hline $\begin{array}{l}\text { PRP platelet } \\
\left(\times 10^{3} / \mu \mathrm{L}\right)\end{array}$ & $492.40 \pm 156.93$ & $618.27 \pm 207.56$ & 0.11 \\
\hline$\%$ platelet increase & $258 \pm 87$ & $330 \pm 156$ & 0.72 \\
\hline $\begin{array}{l}\text { Lysed platelet } \\
\left(\times 10^{3} / \mu \mathrm{L}\right)\end{array}$ & $458.75 \pm 160.11$ & $574.12 \pm 229.02$ & 0.17 \\
\hline Total protein & $9.49 \pm 5.76$ & $7.04 \pm 2.63$ & 0.26 \\
\hline VEGF level (pg/mL) & $103.55 \pm 89.33$ & $406.76 \pm 377.92$ & 0.01 \\
\hline $\begin{array}{l}\text { VEGF per } 1 \times 10^{3} \\
\text { platelet }(\mathrm{fg})\end{array}$ & $0.26 \pm 0.26$ & $0.79 \pm 0.66$ & 0.03 \\
\hline $\begin{array}{l}\text { VEGF per total } \\
\text { protein }(\mathrm{pg} / \mathrm{mg})\end{array}$ & $14.28 \pm 15.15$ & $70.88 \pm 86.65$ & 0.01 \\
\hline
\end{tabular}

PRP, platelet-rich plasma; VEGF, vascular endothelial growth factor.

due to freeze-thawing was also similarly measured.

\section{Statistical analysis}

Numeric data is presented as mean \pm standard deviation. Mean of HbAlc, whole blood platelet, PRP platelet number, total protein and VEGF levels were compared between nondiabetic and diabetic groups. Significance tests were done using individual t-test for normally distributed data and Mann-Whitney $\mathrm{U}$ for non-normally distributed data, with $\mathrm{P}$ value less than 0.05 considered as statistically significant.

\section{Results}

Demographic of patients that participated in this study are presented in Table 1. Most donor ages ranged between 50-75 years old. The mean blood HbA1c level of the diabetic group was statistically significantly higher than in nondiabetic group $(5.58 \% \pm 4.67 \%, 7.06 \% \pm 1.30 \%, \mathrm{P}=0.001)$.

There was no significant difference in platelet counts of whole blood between diabetic and non-diabetic groups. However, diabetic donors had higher platelet numbers than non-diabetic donors in the whole blood samples. There was an average increase of more than $250 \%$ in platelet number after PRP preparation, both in non-diabetic and diabetic groups $(258 \% \pm 87 \%, 330 \% \pm 156 \%, P=0.72)$. We also found that freeze-thawing lysed more than $90 \%$ of the platelet in PRP, regardless of diabetic status $(93 \% \pm 6 \%, 91 \% \pm 14 \%$, $\mathrm{P}=0.32)$. The diabetic PRP showed lower mean total protein but higher VEGF levels relative to non-diabetic PRP. The level of VEGF per total protein in diabetic PRP was significantly higher than non-diabetic PRP. Furthermore, we also observed that lysed platelets from diabetic donors released more VEGF than non-diabetic donors (Table 2).

\section{Discussion}

Foot deformities in conjunction with impaired sensation and infections are the primary cause of foot ulcer formation in diabetic patients. Wound healing processes in diabetic patients are disrupted due to chronic ischemic and inadequate tissue perfusion (14). PRP is well known for its angiogenic potential $(15,16)$, thus it may be beneficial for treatment of DFU. Autologous PRP preparation requires collection of fresh whole blood using vacutainer tubes containing sodium citrate, acid citrate dextrose, citrate phosphate dextrose adenine, or ethylenediaminetetraacetic acid (EDTA), to prevent platelet activation during the preparation process $(17,18)$. To collect concentrated platelets either single- or double-centrifugation requiring centrifugation at slower speeds (generally below $300 \times \mathrm{g}$ ) is used to separate plasma and red blood cell fractions, followed by centrifugation at higher speeds above $300 \times g$ (19). Centrifugation can be done for $5-20$ minutes at $8-20^{\circ} \mathrm{C}$ or at room temperature (19). The final volume of PRP can be adjusted to $3-30 \%$ of initial whole blood volume collected. Overall, PRP preparation is considered simple and can be done in most standard laboratories. However, the composition of PRP needs to be evaluated to ensure it is clinically efficacious.

PRP is composed of a higher concentration of platelets when compared to plasma or whole blood. In this study, platelets in PRP were $250 \%$ more concentrated than whole blood, regardless of donor status. One micro-liter 
of diabetic or non-diabetic derived PRP contained $0.5 \times 10^{6}$ and $0.4 \times 10^{6}$ platelets on average, respectively. For bone and soft tissue regeneration, the optimum platelet concentration in PRP therapy is set at $1 \times 10^{6}$ platelets per micro-liter. However, these general criteria have not been standardized.

PRP has a high concentration of growth factors per milliliter. Transforming growth factor-beta (TGF- $\beta$ ), fibroblast growth factor-2 (FGF-2), platelet-derived growth factors (PDGF-AB), insulin-like growth factor-1 (IGF-1), VEGF, epidermal growth factor (EGF), and hepatocyte growth factor (HGF) are all stored in platelet $\alpha$-granules and released upon platelet activation (20). Platelets can be activated through chemical agents, such as thrombin or calcium chloride solution, or mechanically activated using physical friction or freezethawing techniques (20). Freeze-thawing was applied in this study to store PRP prior to analysis. Storing PRP at $-80{ }^{\circ} \mathrm{C}$ followed by thawing resulted in the lysis of more than $90 \%$ of platelets in samples, with diabetic PRP fractions containing VEGF at $406.76 \pm 377.92 \mathrm{pg} / \mathrm{mL}$, almost four times higher than VEGF in non-diabetic PRP fractions $(103.55 \pm 89.33 \mathrm{pg} / \mathrm{mL})$. Interestingly, the increased VEGF concentrations in diabetic PRP was not directly linked to the significant increased platelet content neither in blood or PRP. These results suggested that platelets from diabetic patients contained higher VEGF than non-diabetic platelets, supporting previously reported results.

Diabetes-related vascular complication is closely related to the increased aggregation of platelet (21). Platelets in diabetic blood were easier to form an aggregate under the same centrifugation process applied to non-diabetic blood that was seen in this study by higher platelet count in diabetic PRP compared to non-diabetic PRP, yet the blood platelet count of both groups was almost similar. Aggregated platelet releases cytokines and growth factors that activates endothelium (21). Once endothelium is activated, it recruits circulating immune cells and more platelets to adhere result in plaque formation. The rupture of any plaque can damage endothelium causing thrombus formation and vascular occlusion. Vascular occlusion triggers the distal tissue becoming ischemic and hypoxic. Hypoxia condition is a trigger for angiogenesis process by increasing HIF$1 \alpha$ and VEGF production to restore blood flow towards the ischemic tissue (22). Thus, this higher VEGF level in plasma may be reflected as a compensating mechanism for ischemia in diabetic patients (13). An excess of circulating VEGF can be endocytosed by platelets and megakaryocytes causes the higher VEGF content in diabetic platelet (23).
We acknowledge that PRP in this study had lower platelet and VEGF concentrations than in other reported studies (19). In this study, we characterized PRP based on VEGF concentration, because VEGF is a potent angiogenic factor. It has been well known that plasma contains balanced proportions of both anti-angiogenic and angiogenic factors. Studies in patients with both peripheral arterial disease and type 2 diabetes compared to healthy subjects revealed that anti-angiogenic factors soluble VEGF receptor 1 and 2 (sVEGFR1, sVEGFR2) were 2-965 times higher than pro-angiogenic VEGF in all groups (13). sVEGFR are a soluble form of VEGFR that can binds to VEGF and prevent VEGF from interacting on cell membrane expressed VEGFR thereby preventing stimulation of angiogenesis (24). Technically, both platelet and VEGF concentrations per microliter can be increased by increasing the speed of centrifugation, however we did not do so for this study. High speed centrifugation can also concentrate both the pro- and anti-angiogenic factors. In a condition when the anti-angiogenic factors predominate, angiogenesis is suppressed, and it is not expected, particularly for DFU treatment (25).

Another technique used to increase platelet or VEGF concentration in PRP is increasing input whole blood volume and reducing PRP final volumes to concentrate samples. Although reports have shown that there was no significant increase in human endothelial cell proliferation and migration produced by plasma stimulation at concentrations of less than $1 \times 10^{6}$ platelets per micro-liter (26). Therefore, increasing input whole blood volume higher than $24 \mathrm{~mL}$ in order to extract more platelets may not be beneficial. Additionally, in clinical practice all of the PRP extracted will be used for treating the ulcers so concentration through reduction of PRP final volume may not be beneficial.

While changing the input whole blood volumes or final PRP volumes may not provide clinical benefit, modifying techniques to activate platelets is a promising method to increase the release of growth factors from platelets. Freezing PRP samples at $-86{ }^{\circ} \mathrm{C}$ followed by thawing can dramatically lyse the platelets. Growth factor release from platelets decreases stability increasing the potential loss of efficacy (27). Freezing PRP at $-20{ }^{\circ} \mathrm{C}$ can also slowly release the growth factors from lysed platelets, but in our experience, the produced PRP will not be suitable for intravenous infusions as it increases the risk of thrombus formation. Clotting can be done ex-vivo by adding calcium chloride or thrombin into PRP. Combining thrombin and calcium chloride can increase VEGF level in PRP (20). 
However, an effective treatment is not only reliant on the high dose given. To create an effective treatment, the practician should consider the dose, the safety, the technique of administrations and the treatment regime combination. We believe that giving patients PRP both locally and systemically may increase the regenerative effect of PRP. Thus, we further customized our PRP preparation protocol by freezing samples at $-86{ }^{\circ} \mathrm{C}$ to store the PRP prior to analysis or for use in mesenchymal stem cell culture. For clinical application, platelet activation was done chemically to result in a safe PRP product for intravenous administration, while maintaining the growth factors content for regenerative function.

Disturbed angiogenesis in diabetic patients can lead to development of DFU. PRP is safe and feasible in clinical practice for diabetes or non-diabetes treatment. Further studies with regards to the composition of PRP based on its pro- and anti-angiogenic factors should be done to develop the most effective PRP treatment for DFU. We have developed a protocol for PRP sample preparation and have found that diabetic PRP is rich in VEGF making it a potential candidate as treatment for DFU and tissue regeneration. However, thrombus formation that is frequent in vascular diabetic patients should be anticipated if intravenous administration route is applied, e.g. by performing ex vivo fibrin clot formation using activation agent such as calcium chloride. Phase I/II clinical trial should be done to investigate the efficacy of PRP prepared by our protocol in this study for the treatment of DFU.

\section{Conclusions}

PRP from diabetic donors had higher VEGF content making autologous PRP application a promising treatment for DFU. However, this should be investigated another appropriate clinical trial.

\section{Acknowledgments}

None.

\section{Footnote}

Conflicts of Interest: The authors have no conflicts of interest to declare.

Ethical Statement: The authors are accountable for all aspects of the work in ensuring that questions related to the accuracy or integrity of any part of the work are appropriately investigated and resolved. The study was conducted in accordance with protocols reviewed and approved by Health Research Ethics Committee, University of Indonesia and Cipto Mangunkusumo Hospital with issued letter of approval No. 628/UN2.F1/ETIK/2016.

\section{References}

1. Liu J, Qu W, Li R, et al. Efficacy of autologous plateletrich gel in the treatment of deep grade II burn wounds. Int J Clin Exp Med 2018;11:2654-9.

2. Babaei V, Afradi H, Gohardani HZ, et al. Management of chronic diabetic foot ulcers using platelet-rich plasma. J Wound Care 2017;26:784-7.

3. da Silva FA, Rodrigues BL, Huber SC, et al. The use of platelet rich plasma in the treatment of refractory Crohn's disease. Int J Clin Exp Med 2017;10:7533-42.

4. Chakravdhanula U, Anbarasu K, Verma VK, et al. Clinical efficacy of platelet rich plasma in combination with methotrexate in chronic plaque psoriatic patients. Dermatol Ther 2016;29:446-50.

5. Bui K, Duong T, Nguyen N, Nguyen T, et al. Symptomatic knee osteoarthritis treatment using autologous adipose derived stem cells and plateletrich plasma: A clinical study. Biomed Res Ther 2014;1:2-8.

6. Chawla S. Split face comparative study of microneedling with prp versus microneedling with vitamin $\mathrm{c}$ in treating atrophic post acne scars. J Cutan Aesthet Surg 2014;7:209-12.

7. Bertrand-Duchesne MP, Grenier D, Gagnon G. Epidermal growth factor released from platelet-rich plasma promotes endothelial cell proliferation in vitro. J Periodontal Res 2010;45:87-93.

8. Xian LJ, Chowdhury SR, Saim A, et al. Concentrationdependent effect of platelet-rich plasma on keratinocyte and fibroblast wound healing. Cytotherapy 2015;17:293-300.

9. Van Pham P, Bui KH, Ngo DQ, et al. Activated platelet-rich plasma improves adipose-derived stem cell transplantation efficiency in injured articular cartilage. Stem Cell Res Ther 2013;4:91.

10. Suryani D, Pawitan JA, Lilianty J, et al. Comparison of fetal bovine serum and platelet-rich plasma on human lipoaspirate-derived mesenchymal stem cell proliferation. Med J Indones 2013;22:146-51.

11. Alexiadou K, Doupis J. Management of diabetic foot ulcers. Diabetes Ther 2012;3:4.

12. Wang J, Chen S, Jiang F, et al. Vitreous and plasma 
VEGF levels as predictive factors in the progression of proliferative diabetic retinopathy after vitrectomy. PLoS One 2014;9:e110531.

13. Wieczór R, Gadomska G, Ruszkowska-Ciastek B, et al. Impact of type 2 diabetes on the plasma levels of vascular endothelial growth factor and its soluble receptors type 1 and type 2 in patients with peripheral arterial disease. J Zhejiang Univ Sci B 2015;16:948-56.

14. Noor S, Zubair M, Ahmad J, et al. Diabetic foot ulcer--A review on pathophysiology, classification and microbial etiology. Diabetes Metab Syndr 2015;9:192-9.

15. Bir SC, Esaki J, Marui A, et al. Angiogenic properties of sustained release platelet-rich plasma: Characterization invitro and in the ischemic hind limb of the mouse. J Vasc Surg 2009;50:870-879.e2.

16. Kurita J, Miyamoto M, Ishii Y, et al. Enhanced vascularization by controlled release of platelet-rich plasma impregnated in biodegradable gelatin hydrogel. Ann Thorac Surg 2011;92:837-44.

17. Ali SF. Platelet activation in stored platelet concentrates: comparison of two methods preparation. J Blood Dis Trans 2011;2:107.

18. do Amaral RJ, da Silva NP, Haddad NF, et al. Platelet-rich plasma obtained with different anticoagulants and their effect on platelet numbers and mesenchymal stromal cells behavior in vitro. Stem Cells Int 2016;2016:7414036.

19. Dhurat R, Sukesh MS. Principles and methods of preparation of platelet-rich plasma: A review and author's

doi: $10.21037 /$ sci.2019.10.02

Cite this article as: Karina, Wahyuningsih KA, Sobariah S, Rosliana I, Rosadi I, Widyastuti T, Afini I, Wanandi SI, Soewondo P, Wibowo H, Pawitan JA. Evaluation of platelet rich plasma from diabetic donors shows increased platelet vascular endothelial growth factor release. Stem Cell Investig 2019;6:43. perspective. J Cutan Aesthet Surg 2014;7:189-97.

20. Hosny N, Goubran F, BadrEldin Hasan B, et al. Assessment of Vascular Endothelial Growth Factor in Fresh versus Frozen Platelet Rich Plasma. J Blood Transfus 2015;2015:706903.

21. Huang D, Refaat M, Mohammedi K, et al. Macrovascular complications in patients with diabetes and prediabetes. BioMed Res Int 2017;2017:7839101.

22. Ando J, Yamamoto K. Vascular mechanobiology: endothelial cell responses to fluid shear stress. Circ J 2009;73:1983-92.

23. Salven P, Orpana A, Joensuu A. Leukocytes and platelets of patients with cancer contain high levels of vascular endothelial growth factor-1. Clin Cancer Res 1999;5:487-91.

24. Failla CM, Carbo M, Morea V. Positive and negative regulation of angiogenesis by soluble vascular endothelial growth factor receptor-1. Int J Mol Sci 2018;19:1306.

25. Cheng R, Ma JX. Angiogenesis in Diabetes and Obesity. Rev Endocr Metab Disord 2015;16:67-75.

26. Rughetti A, Giusti I, D'Ascenzo S, et al. Platelet gelreleased supernatant modulates the angiogenic capability of human endothelial cells. Blood Transfus 2008;6:12-7.

27. Nugraha Y, Sari P, Purwoko RY, et al. Effect of lysed platelet count in platelet concentrates on various growth factor levels after freeze thaw cycles. Int J PharmTech Res 2014;6:2036-42. 